\title{
Contenido de azufre, aluminio, hierro y manganeso foliar en especies vegetales cultivadas en suelo sulfatado ácido
}

\section{Content of foliar sulfur, aluminum, iron and manganese in plant species grown in acid sulfate soil}
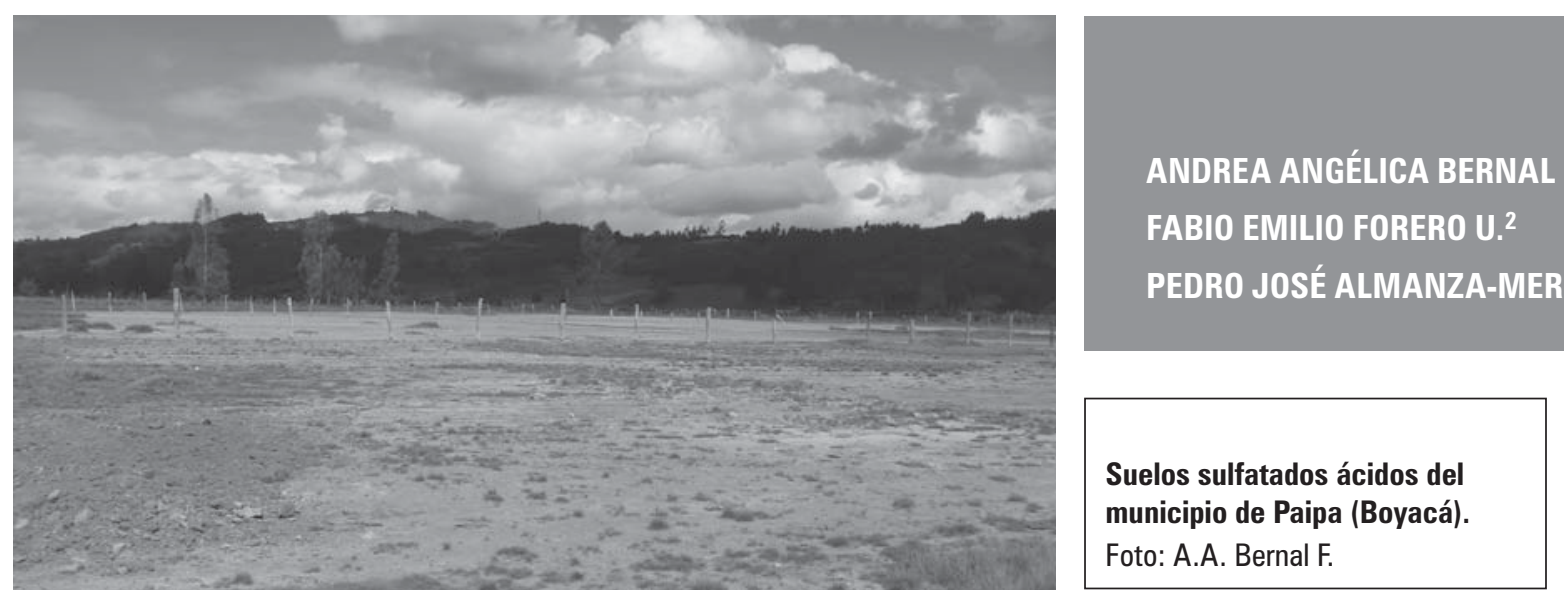

\section{RESUMEN}

Los suelos sulfatados ácidos (SSA) se caracterizan por contener altas cantidades de minerales ricos en hierro y azufre, que al ser aireados, se oxidan y forman horizontes extremadamente ácidos, lo cual afecta el crecimiento de las plantas y genera una superficie escaldada. La siembra de especies vegetales como cultivos de cobertura, se plantea como una práctica ambiental que puede ser implementada en estos suelos. En este estudio, se realizaron análisis foliares a tres especies vegetales, remolacha forrajera (Beta vulgaris L.), nabo forrajero (Brassica rapa L.) y rábano forrajero (Raphanus sativus L.) sembradas en un SSA, contrastado con un suelo no sulfatado, con el fin de evaluar excesos o deficiencias de azufre (S), aluminio (Al), hierro (Fe) y manganeso $(\mathrm{Mn})$ en plantas sembradas en un SSA proveniente del municipio de Paipa (Boyacá, Colombia). El ensayo se realizó bajo cubierta de plástico, sembradas las plantas en bolsas plásticas negras de $5 \mathrm{~kg}$ y, al finalizar su periodo vegetativo, se midieron las concentraciones foliares de los elementos propuestos. Los resultados indicaron que las plantas sembradas en el SSA presentaron mayores contenidos foliares de S, Al, Fe y Mn, con respecto a los registrados en las plantas del suelo no sulfatado, probablemente debido a una mayor capacidad de acumulación o extracción por parte de las especies, o a una mayor disponibilidad de elementos en el SSA.

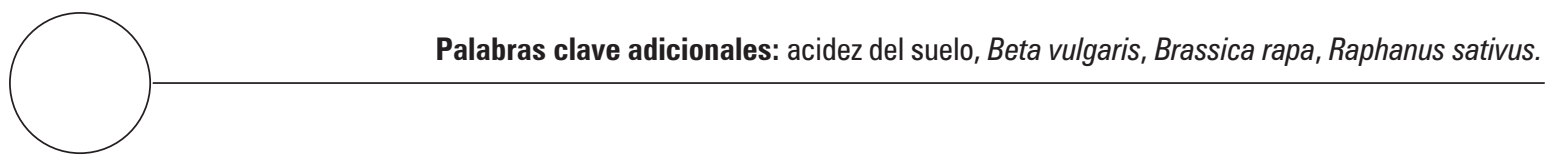

\footnotetext{
Programa de Ingeniería Ambiental, Universidad de Boyacá, Tunja (Colombia).

Facultad de Ciencias Agropecuarias, Universidad Pedagógica y Tecnológica de Colombia (UPTC), Tunja (Colombia).

Autor para correspondencia. anbernal@gmail.com
} 


\section{ABSTRACT}

Acid sulfate soils (ASS) are characterized by having high amounts of iron and sulfur minerals, which, in the presence of air, are oxidized and form sulfuric horizons that are extremely acidic, which affects the growth of plants and generates a scalded surface. Using plants as cover crops is proposed as an environmental practice that can be implemented in these soils. In this study, a leaf analysis of three plant species, fodder beet (Beta vulgaris L.), forage turnip (Brassica rapa L.) and fodder radish (Raphanus sativus L.) planted in an ASS, was contrasted with that of a non-sulfated soil in order to evaluate the excesses or deficiencies of sulfur (S), aluminum (Al), iron (Fe) and manganese ( $\mathrm{Mn}$ ) in plants sown in an ASS of Paipa (Boyacá). The plants were hold under greenhouse plastic cover, in $5 \mathrm{~kg}$ black plastic bags, and, at the end of their growing season, the foliar concentrations of the elements proposed were measured. The results indicated that the plants sown in the ASS had higher foliar contents of S, Al, Fe and Mn than the plants in the non-sulfated soil, probably due to an increase or removal of the storage capacity by the species or increased availability of the elements in the ASS.

Additional key words: soil acidity, Beta vulgaris, Brassica rapa, Raphanus sativus.

Fecha de recepción: 28-09-2015

Aprobado para publicación: 30-11-2015

INTRODUCCIÓN

El Distrito de Riego del Alto Chicamocha (DRACH) en el Departamento de Boyacá es uno de los más importantes del oriente colombiano (Rincón et al., 2008); en él se encuentran alrededor de 3.000 ha de suelos sulfatados ácidos (SSA) que por sus características físicas, químicas y biológicas, son atípicos en la región (Bello y Gómez, 2001; Pardo et al., 2009). Estos suelos se caracterizan por ser sistemas dinámicos con una degradación continua a causa de procesos de acidificación actual severa, producto de la oxidación primaria de sulfuros de hierro y la generación de ácido sulfúrico $\left(\mathrm{H}_{2} \mathrm{SO}_{4}\right)$ (Gómez, 2006). Este ácido deteriora severamente el ambiente y al moverse a través del suelo, libera hierro, aluminio o manganeso y, en algunos casos también disuelven elementos como arsénico y zinc, mezcla que puede volverlos extremadamente ácidos y tóxicos, ante lo cual pocas plantas pueden sobrevivir, generándose en muchos casos una superficie escaldada (Sammut y Lines, 2000).

En los últimos años algunas investigaciones se han encaminado a recuperar este tipo de sue- los (Ercoli et al., 1999), y como consecuencia de ello se han utilizado una serie de tecnologías de remediación convencional; sin embargo, la mayoría son costosas de implementar y perturban el medio ambiente (Ercoli et al., 1999; Reddy et al., 1999); en este sentido, el manejo de los SSA se ve afectado por los altos costos que demanda su recuperación (Rincón et al., 2008). Dentro de las prácticas de manejo recomendadas están la siembra de especies tolerantes, la incorporación de abonos vegetales al suelo para el mantenimiento de un nivel adecuado de materia orgánica, o la labranza mínima y cero, entre otras, las cuales tienden a disminuir los efectos negativos generados en los suelos (Honorato y Bonomelli, 2002). La tolerancia de las plantas sembradas en estos suelos está dada por la capacidad para resistir la acumulación de elevados niveles de ciertos elementos en sus tejidos, basada en estrategias fisiológicas que adoptan para resistir sus efectos tóxicos (López, 2007); adicionalmente, la siembra de especies vegetales como cultivos de cobertura generalmente no altera estos ecosistemas, ya que es una técnica pasiva, amigable y sosteni- 
ble con el medio ambiente, la cual se puede utilizar junto con métodos mecánicos tradicionales o en algunos casos en reemplazo de ellos. No obstante, esta metodología debe realizarse de forma controlada para evitar el paso de elementos en exceso a la cadena trófica.

En este contexto, el análisis foliar se considera una referencia indispensable para determinar tanto las necesidades de nutrientes que deben ser aplicados, como los estados de deficiencia o exceso de macro y microelementos en los cultivos; de acuerdo con Legaz et al. (1995), esto se debe a que indican el grado de absorción de los mismos por la planta, puesto que las hojas son muy sensibles a los cambios en la composición del medio nutritivo. De igual forma, son un complemento indispensable a los análisis de suelo y necesarios para lograr un adecuado diagnóstico (Legaz et al., 1995). Este tipo de análisis ha adquirido una importancia cada vez mayor; sin embargo, a pesar de ello y a su amplio uso en otros países, en muchos cultivos tecnificados de Colombia no se emplea esta técnica (Osorio, 2012). De acuerdo con lo anteriormente expuesto, en esta investigación se realizaron análisis foliares de tres especies vegetales sembradas en SSA y suelo no sulfatado ácido, para evaluar y comparar los excesos o deficiencias de algunos minerales que hacen parte de la problemática en los SSA, como el azufre, aluminio, hierro y manganeso.

\section{MATERIALES Y MÉTODOS}

\section{Localización y muestreo}

El SSA se colectó en el sector Varguitas del municipio de Paipa (Boyacá, Colombia) ubicado a una altitud promedio de $2.500 \mathrm{msnm}$, con coordenadas 54'51"N y 73³"29' W, debido a los reportes acerca de la existencia de suelos con características de acidez extrema en la zona (GISSAT, 2006), la cual forma parte del DRACH en el departamento de Boyacá. Se tomó muestra de $1 \mathrm{~kg}$ de suelo según la metodología planteada por Bautista et al. (2011) para llevar a cabo su caracterización de acuerdo con las recomendaciones estipuladas por el IGAC (2006). Asimismo, se recolectaron $135 \mathrm{~kg}$ de suelo a una profundidad no superior a $20 \mathrm{~cm}$, los cuales fueron trasladados al Jardín Botánico de la Universidad Pedagógica y Tecnológica de Colombia (Tunja), donde se recolectaron adicionalmente $135 \mathrm{~kg}$ de suelo no sulfatado provenientes de las actividades de siembra en el Jardín, para realizar el montaje de las unidades experimentales (UE). El proceso de análisis de muestras se realizó en el laboratorio de docencia de suelos de la Facultad de Ciencias Agropecuarias de la Universidad Pedagógica y Tecnológica de Colombia (UPTC Tunja).

\section{Diseño experimental}

Se realizó el montaje bajo cubierta de plástico, dentro del Jardín Botánico de la UPTC a una altitud de $2.680 \mathrm{msnm}$. Se utilizó un diseño experimental completamente al azar con seis tratamientos, [T1]: suelo no sulfatado + remolacha forrajera (B. vulgaris.); [T2]: suelo no sulfatado + nabo forrajero (B. rapa); [T3]: suelo no sulfatado + rábano forrajero ( $R$. sativus); [T4]: suelo sulfatado ácido + remolacha forrajera (B. vulgaris); [T5]: suelo sulfatado ácido + nabo forrajero $(B$. rapa) y [T6]: suelo sulfatado ácido + rábano forrajero ( $R$. sativus), cada uno con tres repeticiones para un total de 18 UE. Cada UE estuvo compuesta por tres bolsas negras de polietileno para vivero con capacidad para $5 \mathrm{~kg}$ de suelo.

Los resultados arrojados en la caracterización química de los dos tipos de suelo (medición cero) se muestran en la tabla 1, a partir de los cuales se realizaron los cálculos de las cantidades necesarias de enmienda a emplear como correctivo. En el SSA se agregaron $20 \mathrm{t} \mathrm{ha}^{-1}$ de cal dolomita y en el suelo no sulfatado se adicionaron $8,8 \mathrm{t} \mathrm{ha}^{-1}$ de cal agrícola. Luego de un periodo de reacción de la enmienda de $60 \mathrm{~d}$ se sembraron 4 semillas de las especies vegetales por bolsa; se llevó a cabo riego por aspersión durante 30 min aproximadamente con un intervalo de dos días. Al finalizar el periodo vegetativo (120 d después de la siembra), se 
Tabla 1. Caracterización química inicial (medición cero) de los dos tipos de suelo.

\begin{tabular}{|l|c|c|c|c|c|}
\hline \multirow{2}{*}{\multicolumn{1}{c}{ Tipo de suelo }} & \multirow{2}{*}{$\mathrm{pH}$} & $\mathrm{Al}$ & $\mathrm{S}$ & \multicolumn{2}{c|}{$\mathrm{Fe}$} \\
\cline { 3 - 7 } & & $\mathrm{cmol}_{\mathrm{c}} \mathrm{kg}^{-1}$ & & \multicolumn{2}{|c|}{$\mathrm{mg} \mathrm{L}^{-1}$} \\
\hline Suelo sulfatado ácido (SSA) & 3,15 & 12,6 & 167,0 & 115,47 & 3,50 \\
\hline Suelo no sulfatado (SN) & 5,22 & $\mathrm{ND}$ & 30,8 & 3,56 & 4,84 \\
\hline
\end{tabular}

ND: no determinado

recolectó la producción para realizar los análisis foliares de S, Al, Fe y Mn en cada una de las especies en estudio.

\section{Análisis de laboratorio}

Los extractos para los análisis foliares se realizaron en el Laboratorio de Nutrición Animal de la Facultad de Ciencias Agropecuarias de la UPTC, de acuerdo con la metodología planteada por Carrillo et al. (1994). La cuantificación de Fe, Al y Mn se llevó a cabo por el método de Espectofotometría de Absorción Atómica (EAA) (Carrillo et al., 1994) y el S mediante la técnica de colorimetría (IGAC, 2006), en el Laboratorio de Diagnóstico de Suelos y Aguas de la Facultad de Ciencias Agropecuarias de la UPTC.

\section{Análisis estadístico}

Se realizó un análisis de varianza (Anova) para determinar diferencias estadísticas entre tratamientos, y se utilizó la prueba de comparación de medias Tukey con una confiabilidad del 95\%, con el programa estadístico SAS v. 9.1.

\section{RESULTADOS Y DISCUSIÓN}

\section{Contenido de azufre}

Se presentaron diferencias estadísticas significativas $(P \leq 0,05)$ en las concentraciones de $S$ foliar. La remolacha presentó contenidos estadísticamente similares en los dos tipos de suelo; sin embargo, se observó un valor mayor de la especie en el SSA. Por su parte, el nabo y rábano en SSA mostraron niveles superiores a los obtenidos en el suelo no sulfatado, lo cual podría indicar que el tipo de suelo posiblemente influyó en la concentración de $\mathrm{S}$ foliar en las especies propuestas (figura 1). Kalra (1998) e IGAC (2013) reportan que la concentración normal de $S$ en tejido foliar se encuentra en un rango entre 0,25 y $1,0 \%$, los cuales son superiores a los obtenidos en este estudio en los seis tratamientos, lo que indicaría una concentración baja de este elemento debido a que se registran valores inferiores al 0,16\%. Por otra parte, Barbazán (1998) menciona valores normales de contenido de $\mathrm{S}$ en las plantas de entre 0,1 y $0,4 \%$, dentro del cual se encuentra la concentración obtenida en las plantas de nabo en el SSA, mientras que los cinco tratamientos restantes se encuentran por debajo del mismo.

Para el caso particular de las especies propuestas, Reid et al. (1994) registraron un contenido de $S$ de $0,57 \%$ en hojas de nabo forrajero bajo condiciones de suelo no degradado, 90 d después de la siembra, los cuales no coinciden con los obtenidos en las plantas de los dos tipos de suelo; sin embargo, debe tenerse en cuenta que la cosecha en este estudio se realizó a los 120 d, lo que pudo influir posiblemente en las concentraciones registradas de este elementos en las plantas. En cuanto al comportamiento del $\mathrm{S}$ foliar en las especies sembradas en el SSA. De manera general, Reid et al. (1994) manifiestan que la cantidad de $S$ existente en cada planta varía dentro de límites relativamente amplios ( 0,1 y $1 \%$ ), los cuales dependen no solo del contenido en el suelo, sino también de las necesidades fisiológicas propias de cada especie; asimismo, afirman que las con- 


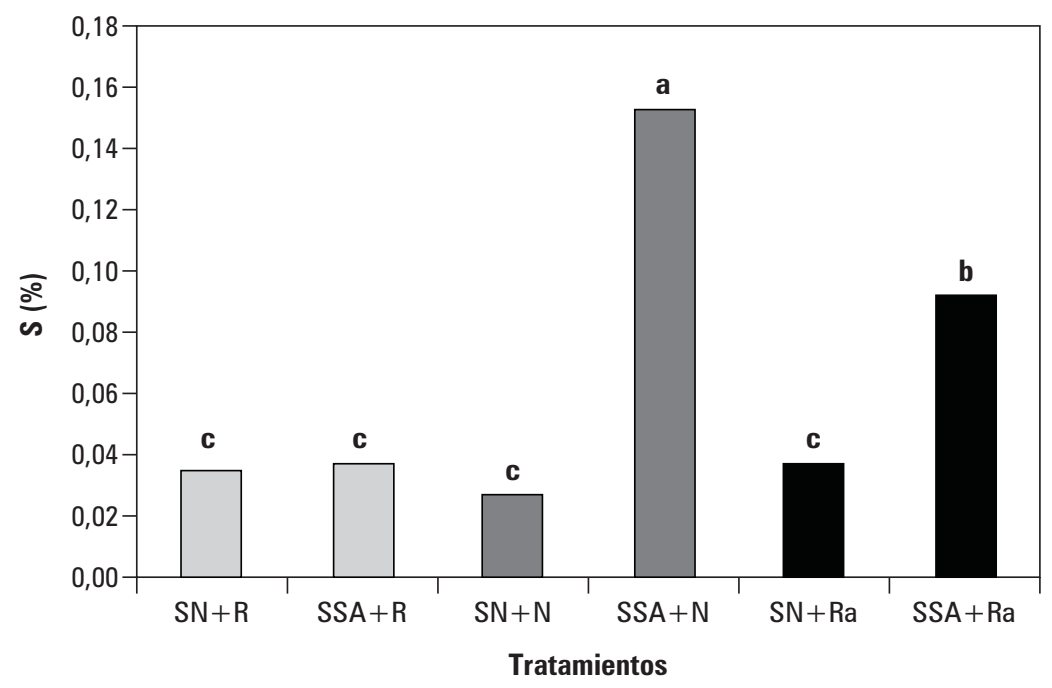

Figura 1. Análisis de contenidos foliares de azufre. SSA: suelo sulfatado ácido; SN: suelo no sulfatado; R: remolacha forrajera; N: nabo forrajero; Ra: rábano forrajero.

Promedios con letras distintas, en la misma serie, indican diferencia significativa según la prueba de Tukey $(P \leq 0,05)$.

centraciones más altas pueden encontrarse en leguminosas y crucíferas, lo que se evidenció en este estudio con el nabo y el rábano. Por su parte, Navarro y Navarro (2003) explican que el S reducido puede ser reoxidado a $\mathrm{SO}_{4}^{-2}$ y permanecer en la planta como reserva, forma bajo la cual se halla distribuido con bastante regularidad en partes vitales como raíces, tallos, hojas, etc.

\section{Contenido de aluminio}

Se presentaron diferencias estadísticas significativas $(P \leq 0,05)$ en las concentraciones de $\mathrm{Al}$ foliar. La remolacha registró los contenidos más altos con un valor de $68,04 \mathrm{mg} \mathrm{L}^{-1}$, comportamiento similar al obtenido en el rábano; por su parte, el nabo mostró contenidos mayores en el suelo no sulfatado (figura 2). De acuerdo con los resultados obtenidos, en la remolacha y el rábano se presentaron variaciones expresadas en mayores contenidos de Al foliar en las plantas del SSA, posiblemente por efecto del tipo de suelo. Según los Laboratorios A-L de México (2011) el Al foliar puede encontrarse en un rango normal entre 0 y $251 \mathrm{mg} \mathrm{L}^{-1}$. De acuerdo con esto, los resultados de los análisis realizados en los seis tratamientos muestran que su concentración se encuentra dentro del rango. Por otra parte, Kalra (1998) aunque no propone rangos deficientes, normales o excesivos, menciona que el contenido medio que se espera ocurra en el tejido foliar puede ser de $80 \mathrm{mg} \mathrm{L}^{-1}$, ante lo cual se puede notar que el Al foliar en los seis tratamientos en este estudio no supera dicho valor.

De forma general, Navarro y Navarro (2003) sugieren que aunque el $\mathrm{Al}$ no es considerado un elemento esencial en las plantas, es benéfico para el desarrollo de determinados cultivos debido a que se cree que pueden estimular la absorción o transporte de otros elementos fundamentales que se encuentran en proporción limitada, o bien inhibir la absorción de otros que se hallen en exceso; así mismo, señalan que incluso las plantas toman ciertos elementos que no se encuentran comúnmente en la naturaleza en cantidades apreciables si están a su alcance, como en el caso del aluminio, el cual es muy abundante en la 


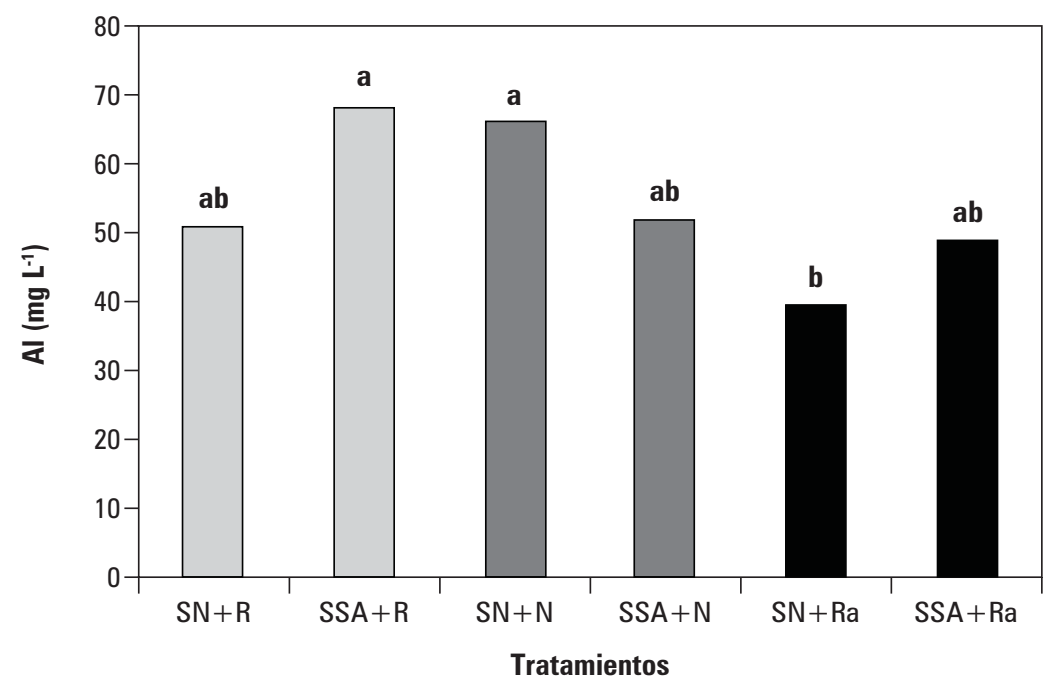

Figura 2. Análisis de contenidos foliares de aluminio. SSA: suelo sulfatado ácido; SN: suelo no sulfatado; R: remolacha forrajera; N: nabo forrajero; Ra: rábano forrajero.

Promedios con letras distintas, en la misma serie, indican diferencia significativa según la prueba de Tukey $(P \leq 0,05)$

corteza terrestre y es absorbido por muchas especies lo que causa alteraciones en determinados órganos de la misma. Al respecto, Cameron et al. (1986) expresan que los síntomas de fitotoxicidad por $\mathrm{Al}$ no dependen únicamente de una concentración crítica del elemento en el sustrato, aunque su presencia es un factor muy importante; igualmente, Gallardo et al. (1999) y Casierra (2001) exponen que características del medio como el $\mathrm{pH}$, la formación de precipitados insolubles, los efectos protectores de iones, la fuerza iónica de la solución, la presencia de quelantes o el genotipo de la planta, pueden también actuar en la modificación de la respuesta de las plantas.

Otros autores, como Kinraide (1991) y Casierra y Niño (2007), manifiestan que muchos de estos factores se pueden comprender mejor si se tienen en cuenta los efectos que causa la especiación de Al, la cual varía de acuerdo al pH. Según Gallardo et al. (2005) las formas tóxicas del Al son función del pH y afectan en distinto grado las especies vegetales y cultivares dentro de una misma especie.

\section{Contenido de hierro}

Se presentaron diferencias estadísticas significativas $(P \leq 0,05)$ en las concentraciones de Fe foliar. Las concentraciones de Fe presentaron contenidos superiores en las plantas de remolacha y rábano sembrados en el SSA con respecto a los registrados en el suelo no sulfatado, mientras el nabo mostró valores estadísticamente similares en los dos tipos de suelo (figura 3). Al igual que el caso del $\mathrm{Al}$, se presentaron mayores contenidos de Fe foliar en las plantas de remolacha y rábano del SSA, debido posiblemente al tipo de suelo. El IGAC (2013) y Kalra (1998) plantean que el Fe foliar puede encontrarse entre 60 y $500 \mathrm{mg} \mathrm{L}^{-1}$; de acuerdo con estos reportes los tratamientos evaluados presentarían valores bajos del mismo. Al respecto, Kalra (1998) sugiere un contenido deficiente por debajo de $50 \mathrm{mg} \mathrm{L}^{-1}$, lo cual indicaría una insuficiencia en los tratamientos, excepto en la remolacha del SSA. Sin embargo, en contraste con lo expuesto, los Laboratorios A-L de México (2011) señalan un rango normal entre 0 y $251 \mathrm{mg} \mathrm{L}^{-1}$, dentro del cual se encuentran los valores registrados en la to- 
talidad de tratamientos. Por su parte, Navarro y Navarro (2003) expresan que normalmente en los tejidos las concentraciones de Fe foliar varía desde 25 a más de $250 \mathrm{mg} \mathrm{L}^{-1}$ de acuerdo con la parte de la planta que se considere, y de la especie; asimismo, exponen que en las hojas, concretamente en los cloroplastos, se encuentra la mayor parte del mismo.

En cuanto a los estudios encontrados sobre los contenidos de Fe en las especies vegetales, en suelos no degradados, Francisco (2010) reporta concentraciones de 2,70 mg y Arias (2009) valores promedio entre 0,01 y $0,24 \mathrm{mg} / 100 \mathrm{~g}$, los cuales son considerablemente superiores a los obtenidos en este estudio; de igual forma, Arias (2009) encontró un contenido de Fe de 0,9 $\mathrm{mg} / 100 \mathrm{~g}$ en la remolacha, el cual es significativamente menor al registrado en este estudio. De acuerdo con Follet et al. (1981), y Navarro y Navarro (2003), el Fe es altamente disponible en un $\mathrm{pH}$ inferior a 5, como en los SSA, e indican que los micronutrientes son siempre absorbidos en cantidades cien a mil veces inferiores a las de los macronutrientes; asimismo, exponen que en el caso del Fe, aparte de la función absorbente realizada por las raicillas de las plantas, estas segregan sustancias dotadas de cierto carácter ácido que les permite solubilizar, en parte, compuestos difícilmente solubles situados en sus cercanías, tales como óxidos de Fe y Mn, entre otros.

Navarro y Navarro (2003) consideran que las plantas pueden captar este elemento por interacción directa de la superficie radicular con las partículas del suelo. Igualmente, sugieren que las sustancias con estructura quelante son excretadas por las raíces, las cuales pueden originar quelatos de Fe que son absorbidos íntegramente por las mismas; sin embargo, expresan que esta capacidad está limitada a determinadas zonas de la raíz y no a todas las especies vegetales, lo cual puede explicar, en parte, el hecho de que haya plantas más sensibles que otras a la falta o escasez de Fe asimilable en el suelo.

\section{Contenido de manganeso}

Se presentaron diferencias estadísticas significativas $(P \leq 0,05)$ en las concentraciones de $\mathrm{Mn}$

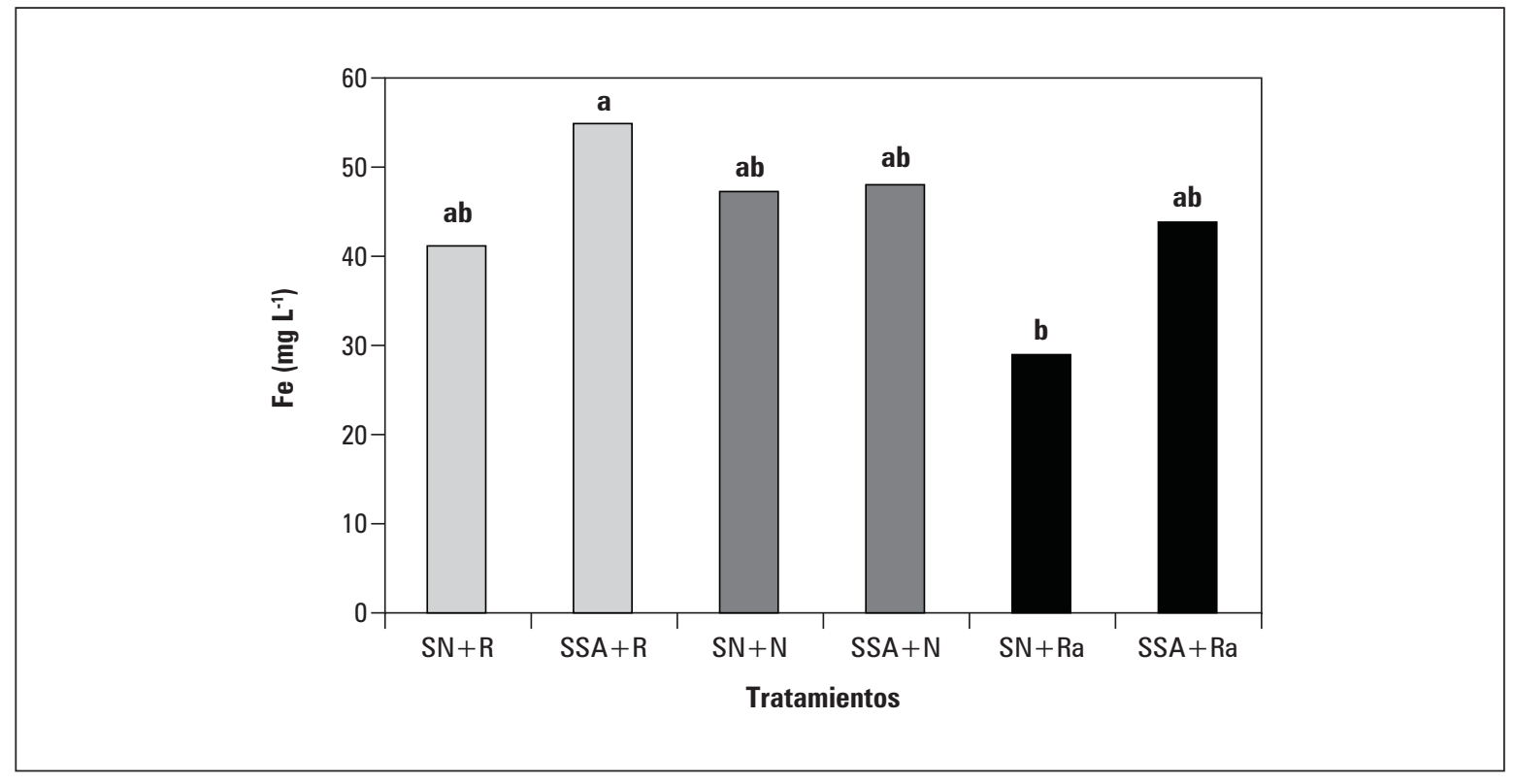

Figura 3. Análisis de contenidos foliares de hierro. SSA: suelo sulfatado ácido; SN: suelo no sulfatado; R: remolacha forrajera; N: nabo forrajero; Ra: rábano forrajero.

Promedios con letras distintas, en la misma serie, indican diferencia significativa según la prueba de Tukey $(P \leq 0,05)$. 
foliar. La remolacha de los dos tipos de suelo presentó los valores más altos entre tratamientos con una concentración notablemente superior en el SSA (figura 4). Aunque no se presentaron diferencias estadísticamente significativas en las concentraciones de $\mathrm{Mn}$ en las plantas de nabo y rábano sembradas en los dos tipos de suelo, se puede notar que se registraron valores más altos en el SSA, en comparación al no sulfatado. De acuerdo con estos resultados, al igual que en los elementos anteriores, se presentaron mayores contenidos de $\mathrm{Mn}$ foliar en las tres especies del SSA, probablemente como consecuencia del tipo de suelo.

En cuanto a los rangos normales del Mn foliar, el IGAC (2013) plantea un rango de 30 a $200 \mathrm{mg}$ $\mathrm{kg}^{-1}$, los Laboratorios A-L de México (2011) entre 30 - 300 mg L-1, mientras Barbazán (1998) sugiere concentraciones entre 20 y $500 \mathrm{mg} \mathrm{L}^{-1}$, y Kalra (1998) manifiesta que puede encontrarse entre 20 y $300 \mathrm{mg} \mathrm{L}^{-1}$, los cuales difieren considerablemente de los valores obtenidos en este estudio; sin embargo, la remolacha de los dos tipos de suelo y, el nabo y rábano del SSA, se encontrarían dentro del rango normal. Los resultados obtenidos en el nabo y rábano del suelo no sulfatado, según Kalra (1998) y Navarro y Navarro (2003), indicarían un contenido deficiente por encontrarse entre 15 y $25 \mathrm{mg} \mathrm{L}^{-1}$.

En referencia a los estudios encontrados de las especies vegetales, Arias (2009) y Francisco (2010) reportaron contenidos promedio de $\mathrm{Mn}$ foliar entre 0,02 y $0,18 \mathrm{mg} / 100 \mathrm{~g}$ y $2.70 \mathrm{mg}$ para el nabo forrajero respectivamente, los cuales son demasiado bajos en comparación a los registrados en esta investigación. Por su parte, Arias (2009) menciona un contenido de Mn foliar en la remolacha de $10 \mathrm{mg} / 100 \mathrm{~g}$, el cual, de igual forma, es bajo en relación a los obtenidos en este estudio. Al respecto Navarro y Navarro (2003) manifiestan que las necesidades cuantitativas de $\mathrm{Mn}$ en las plantas son relativamente pequeñas, pero varían más que en cualquier otro micronutriente y sus contenidos fluctúan de acuerdo con la especie vegetal; sin embargo, los tejidos verdes tienen la mayor concentración de este elemento.

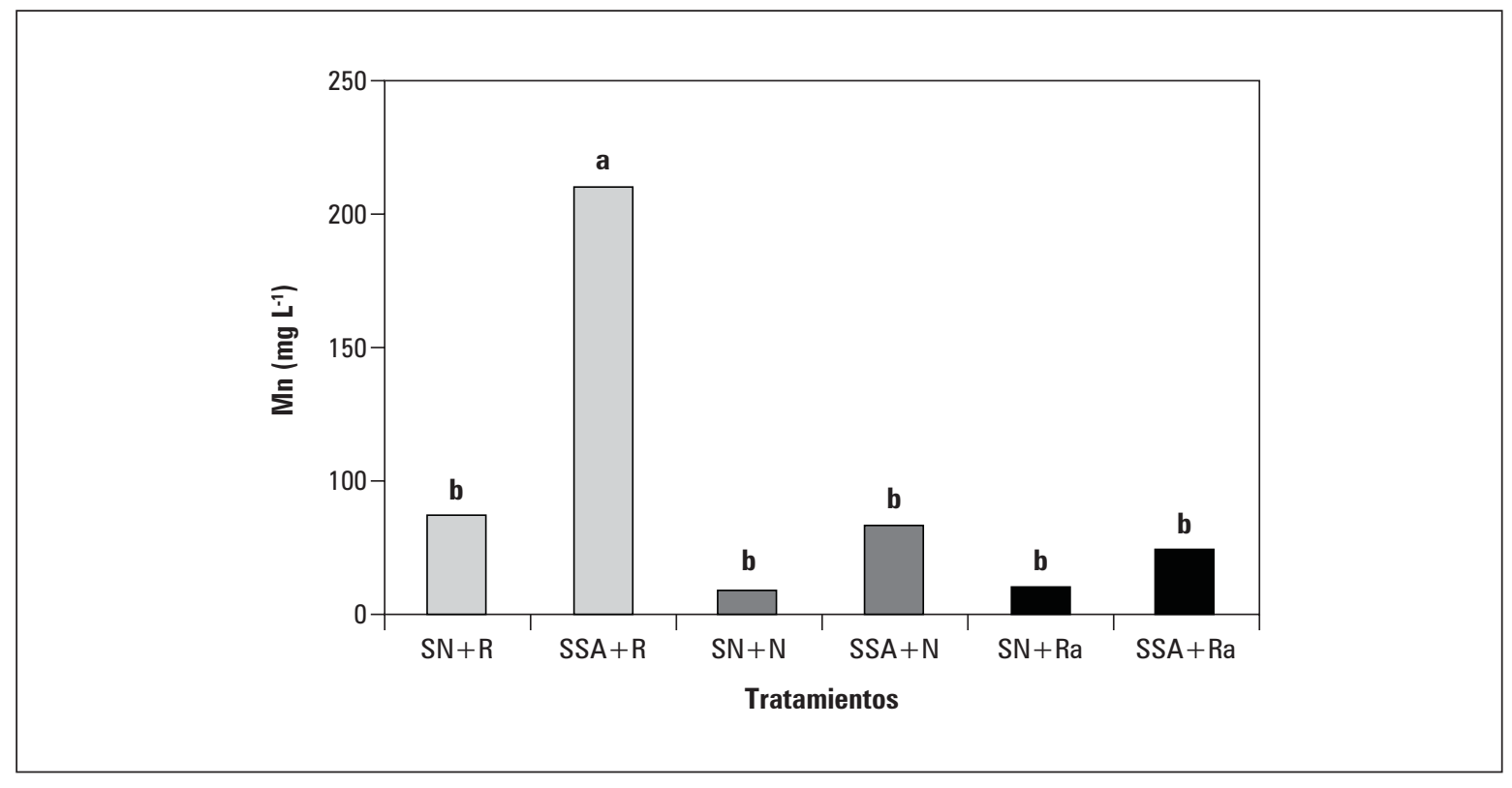

Figura 4. Análisis de contenidos foliares de manganeso. SSA: suelo sulfatado ácido; SN: suelo no sulfatado; R: remolacha forrajera; N: nabo forrajero; Ra: rábano forrajero.

Promedios con letras distintas, en la misma serie, indican diferencia significativa según la prueba de Tukey $(P \leq 0,05)$. 
En cuanto al comportamiento del Mn foliar en las plantas sembradas en SSA, Navarro y Navarro (2003) afirman que las alteraciones por exceso pueden presentarse en este tipo de suelos, donde su disponibilidad está al máximo; de igual forma, estos autores reportan que los niveles de Mn superiores a $100 \mathrm{mg} \mathrm{L}^{-1}$ indican toxicidad, lo cual señala que los seis tratamientos no presentaron esta condición de acuerdo con los resultados obtenidos. Sin embargo, la disponibilidad de este elemento para la planta será elevada en suelos ácidos, debido a la alta solubilidad que presentan los compuestos de $\mathrm{Mn}$ en condiciones de pH bajos (Navarro y Navarro, 2003).

\section{Consideraciones generales}

En general, el contenido de los elementos foliares puede variar dentro de rangos muy amplios y depende de diversos factores como variedad, grado de madurez, tipo de suelo, uso de fertilizantes, intensidad y duración de la luz solar, temperatura o pluviosidad (Costa et al., 2003). De igual forma, Arias (2009) asegura que su concentración depende en gran parte de su contenido en el suelo donde se ha cultivado o producido la planta, lo cual hace que la composición de una misma variedad cultivada en distintas regiones sea diferente en su valor nutritivo. Al respecto, Navarro y Navarro (2003) exponen que las plantas difieren unas de otras en su poder de absorción; igualmente, reportan que especies distintas, cultivadas en un mismo suelo, pueden tener una alimentación mineral diferente bajo el punto de vista cualitativo y cuantitativo, e incluso variedades distintas de una misma especie no actúan del mismo modo.

Hernández (2002) manifiesta que las plantas tienen una capacidad limitada para absorber y acumular elementos no necesarios para su crecimiento, ante lo cual Gutiérrez (1997) señala que las hojas representan uno de los principales con- sumidores de los nutrientes minerales del suelo extraídos por los cultivos; sin embargo, el reciclaje de alguno de ellos durante la senescencia de las hojas o la reproducción es muy reducido. Por su parte, Sánchez de la Puente (1984) afirma que no es posible hablar de contenidos estáticos normales de los nutrientes minerales, porque la planta no presenta en realidad niveles fijos, sino que varían en cada circunstancia. Por esta razón, se presenta dificultad en la interpretación de los resultados analíticos, ya que los métodos son numerosos, las teorías se multiplican y no se prevé un resultado inmediato, además, independientemente de la metodología que pueda aplicarse, no existen niveles óptimos de forma absoluta sino solo relativos según las condiciones determinadas o momentos fisiológicos (Sánchez de la Puente, 1984).

\section{CONCLUSIONES}

La remolacha forrajera presentó contenidos foliares más altos de Al, Fe y Mn en las plantas sembradas en el SSA, mientras que el S mostró un comportamiento similar en los dos tipos de suelo; el nabo forrajero evidenció un contenido de $\mathrm{S}$ foliar considerablemente superior, alto en $\mathrm{Mn}$, similar en Fe y menor en $\mathrm{Al}$ en las plantas del SSA, en comparación con los del no sulfatado. El rábano forrajero presentó contenidos de $\mathrm{S}$, $\mathrm{Al}, \mathrm{Fe}$ y $\mathrm{Mn}$ foliar superiores en las plantas del SSA, esto probablemente debido a una mayor extracción, capacidad mayor de acumulación por parte de las plantas, o a una mayor disponibilidad de elementos en el SSA.

Se presentaron dificultades en cuanto a la interpretación de los análisis foliares, debido a que existen varios métodos y teorías; además, no existen niveles óptimos de forma absoluta, sino solo relativos, de acuerdo con especies vegetales, momentos fisiológicos y condiciones climáticas y ambientales determinadas, entre otros. 


\section{AGRADECIMIENTOS}

Los autores expresan su agradecimiento al médico veterinario Carlos Rodríguez y a los auxiliares del Laboratorio de Nutrición Animal de la Uni- versidad Pedagógica y Tecnológica de Colombia, por la colaboración recibida en la elaboración de los extractos vegetales.

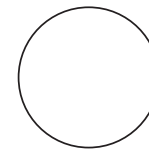

Arias, M. 2009. Caracterización físicoquímica y sensorial de nabiza y grelo (Brassica rapa L.). Tesis doctoral. Departamento de Química Analítica, Universidad de Santiago de Compostela, Santiago de Compostela, España.

Barbazán, M. 1998. Análisis de plantas y síntomas visuales de deficiencia de nutrientes. Informe de asistente de fertilidad de suelos. Facultad de Agronomía, Universidad de la República, Montevideo.

Bautista, Z., G. Delfín y J. Palacio. 2011. Técnicas de muestreo para manejadores de recursos naturales. $2^{a}$ ed. Centro de Investigaciones en Geografía Ambiental, Instituto de Geografía Universidad Nacional Autónoma de México (UNAM), México D.C.

Bello, F. y M. Gómez. 2001. Recuperación de suelos sulfatado ácidos en área del distrito de riego del Alto Chicamocha Tibasosa (Boyacá). Trabajo de grado. Facultad de Ciencias Agropecuarias, Universidad Pedagógica y Tecnológica de Colombia, Tunja, Colombia.

Cameron, R., G. Ritchie y A. Robson. 1986. Relative toxicities of inorganic aluminium complexes to barley. Soil Sci. Soc. Am. J. 50, 1231-1237.

Casierra, F. y R. Niño. 2007. Solubilidad y reacción del aluminio en el suelo. Ciencia y Agricultura 5(2), 7-17.

Casierra, F. 2001. Fundamentos fisiológicos, bioquímicos y anatómicos del estrés por aluminio en vegetales. Rev. Comalfi 28(2), 8-19.

Carrillo, F., B. Mejía y F. Franco. 1994. Manual de laboratorio para análisis foliares. Centro Nacional de Investigaciones de Café (Cenicafé), Chinchiná, Colombia.

Costa, S., M. Montenegro, T. Arregui, M. Pinto, M. Nazareno y B. Mishima. 2003. Caracterización de acelga fresca de Santiago del Estero (Argentina). Comparación del contenido de nutrientes en hoja

\section{REFERENCIAS BIBLIOGRÁFICAS}

y tallo. Evaluación de los carotenoides presentes. Ciênc. Tecnol. Aliment. 23(1), 33-37. Doi: 10.1590/ S0101-20612003000100008

Ercoli, E., J. Gálvez, M. Di Paola, J. Cantero, S. Videla y C. Medaura. 1999. Biorremediación de suelos altamente contaminados. Expl.-8-EE-03. En: Memorias III Congreso Ingepet 1999, Lima.

Follet, R., L. Murphy y R. Donahue. 1981. Fertilizers and soil amendments. Prentice-Hall Inc., London.

Francisco, M. 2010. Compuestos bioactivos y producción de grelos y nabizas: variación fenotípica y ambiental. Tesis doctoral. Universidad de Vigo, Pontevedra, España.

Gallardo, F., M. Pino, M. Alvear y F. Borie. 2005. Efecto del aluminio en la producción de materia seca y en la actividad nitrato reductasa de dos variedades de trigo, creciendo en soluciones nutritivas. R.C. Suelo Nutr. Veg. 5(1), 30-36.

Gallardo, F., F. Borie, M. Alvear y E. Von Baer. 1999. Evaluation of aluminium tolerance of three cultivars by two short-term screening methods and field experiments. Soil Sci. Plant Nutr. 45(3), 713719. Doi: 10.1080/00380768.1999.10415834

GISSAT (Grupo Interinstitucional de Investigación en Suelos Sulfatados Ácidos Tropicales). 2006 Estudio semidetallado de suelos sulfatados ácidos (Documento 1). Proyecto Colciencias-UPTC. Distrito Riego del Alto de Chicamocha (Boyacá). Universidad Pedagógica y Tecnológica de Colombia, Tunja, Colombia.

Gómez, M. 2006. Génesis de suelos sulfatados ácidos y su relación con el manejo agrícola. Distrito de Riego del Alto Chicamocha, Departamento de Boyacá. Tesis de maestría. Facultad de Agronomía, Universidad Nacional de Colombia, Bogotá.

Gutiérrez, M. 1997. Nutrición mineral de las plantas: avances y aplicaciones. Agron. Costarr. 21(1), 127-137. 
Hernández, R. 2002. Nutrición mineral. En: Botánica online. Departamento de Botánica, Facultad de Ciencias Forestales y Ambientales. Universidad de Los Andes, Mérida, Venezuela.

Honorato, R. y C. Bonomelli. 2002. Suelos degradados y agricultura sustentable. Agronomía y Forestal UC $15,20-24$.

IGAC (Instituto Geográfico Agustín Codazzi). 2013. Consideraciones generales para interpretar análisis químicos de suelos. Laboratorio Nacional de Suelos, Subdirección de Agrología, IGAC, Bogotá.

IGAC (Instituto Geográfico Agustín Codazzi). 2006. Métodos analíticos de laboratorio de suelos. $6^{\mathrm{a}}$ ed. Imprenta Nacional de Colombia, Bogotá.

Kalra, Y. (ed.). 1998. Handbook of reference methods for plant analysis. Press, Taylor \& Francis Group, Boca Raton, FL.

Kinraide, T. 1991. Identity of the rhizotoxic aluminum species. Plant Soil 134, 167-178.

Laboratorios A-L de México. 2011. Análisis foliares. En: https://goo.gl/kgcHhE; consulta: noviembre de 2012.

Legaz, F., M. Serna, P. Ferrer, V. Cebolla y E. Primo. 1995. Análisis de hojas, suelos y aguas para el diagnóstico nutricional de plantaciones de cítricos. Procedimiento de toma de muestras. Generalitat Valenciana, Consellería D'Agricultura, Pesca i Alimentació. Gráficas Fortuny S.L.R., Valencia, España.

López, A. 2007. Biorremediación y fitorremediación en suelos contaminados. Publicaciones Real Academia Nacional de Farmacia, Madrid.
Navarro, S. y G. Navarro. 2003. Química agrícola. El suelo y los elementos químicos esenciales para la vida esencial. $2^{\text {a }}$ ed. Mundi-Prensa, Madrid.

Osorio, N. 2012. El análisis foliar: una poderosa herramienta para diagnosticar el estado nutricional de los cultivos, pasturas y plantaciones. En: Manejo integral del suelo y nutrición vegetal Vol. 1, No. 3. Laboratorio de Suelos, Universidad Nacional de Colombia, Medellín, Colombia.

Pardo, S., H. Suárez y V. Pertuz. 2009. Interacción de los suelos sulfatados ácidos con el agua y sus efectos en la sobrevivencia del bocachico (Prochilodus magdalenae) en cultivo. Rev. Colomb. Cienc. Pecua. $22(4), 619-631$.

Reddy, K., J. Adams y C. Richardson. 1999. Potential technologies for remediation of Brownfields. Practice Periodical of Hazardous, Toxic, and Radioactive Waste Management 3(2), 61-68.

Reid, R., J. Puoli, G. Jungf, J. Cox-Ganser y A. McCoy. 1994. Evaluation of brassicas in grazing systems for sheep: I quality of forage and animal performance. J. Animal Sci. 72, 1823-1831.

Rincón, A., H. Castro y M. Gómez. 2008. Caracterización física de los suelos sulfatados ácidos del Distrito de Riego del Alto Chicamocha (Boyacá) y su aplicación al manejo. Agron. Colomb. 26(1), 134-145.

Sammut, J. y K. Lines. 2000. An introduction to acid sulfate soils. $2^{\text {nd }}$ ed. Part of the National Strategy for Coastal Acid Sulfate Soils. Department of the Environment, Australian Government, Australia.

Sánchez de la Puente, L. 1984. La alimentación mineral de las plantas. Instituto de Recursos Naturales y Agrobiología. Temas de divulgación. En: http:// goo.gl/YUrfvH; consulta: noviembre de 2012. 